\title{
From Farey sequences to resonance diagrams
}

\author{
R. Tomás \\ CERN, CH1211 Geneva 23, Switzerland \\ (Received 6 December 2013; published 29 January 2014)
}

\begin{abstract}
Farey sequences were discovered in the beginning of the nineteenth century proving very useful in number theory. These series are found to be tightly related to tune resonance diagrams in accelerators. So far, no connection has been made between these two fields. This paper aims to serve as a bridge between these two areas of knowledge introducing the concept of resonance sequences for the most efficient exploration of the tune space.
\end{abstract}

DOI: 10.1103/PhysRevSTAB.17.014001

PACS numbers: 41.85.-p, 41.75.-i, 02.10.De

\section{INTRODUCTION}

The Farey sequence $F_{N}$ of order $N$ is the ascending sequence of irreducible fractions between 0 and 1 whose denominators do not exceed $N$ [1]. The Farey sequence of order $N$ can also be described as the sequence of all roots of the set of integral linear polynomials $a x-b$ where $0 \leq b \leq a \leq N$, as stated in [2]. This is exactly the same equation that defines the set of tune resonances of order $N$ or lower in one dimension (assuming, for instance, $x=Q_{x}$ ). Therefore the Farey sequence of order $N$ is identical to the ordered one-dimensional sequence of resonances between 0 and 1. For example, the Farey sequences of order 5 and 6 are given by

$$
\begin{gathered}
F_{5}=\left\{\frac{0}{1}, \frac{1}{5}, \frac{1}{4}, \frac{1}{3}, \frac{2}{5}, \frac{1}{2}, \frac{3}{5}, \frac{2}{3}, \frac{3}{4}, \frac{4}{5}, \frac{1}{1}\right\}, \\
F_{6}=\left\{\frac{0}{1}, \frac{1}{6}, \frac{1}{5}, \frac{1}{4}, \frac{1}{3}, \frac{2}{5}, \frac{1}{2}, \frac{3}{5}, \frac{2}{3}, \frac{3}{4}, \frac{4}{5}, \frac{5}{6}, \frac{1}{1}\right\} .
\end{gathered}
$$

Farey sequences have been deeply studied by mathematicians discovering properties that accelerator physicist should profit from. The most useful properties of the Farey sequences follow: (i) The distance between two consecutive fractions, $\frac{a}{b}$ and $\frac{c}{d}$, of any Farey sequence is equal to $\frac{1}{b d}$. For example, the distance between $\frac{2}{5}$ and $\frac{1}{2}$ in $F_{5}$ is $\frac{1}{2}-\frac{2}{5}=\frac{1}{5 \times 2}=\frac{1}{10}$. (ii) If $\frac{a}{b}, \frac{p}{q}$, and $\frac{c}{d}$ are consecutive fractions in any Farey sequence then

$$
\frac{p}{q}=\frac{a+c}{b+d} .
$$

Published by the American Physical Society under the terms of the Creative Commons Attribution 3.0 License. Further distribution of this work must maintain attribution to the author(s) and the published article's title, journal citation, and DOI. (iii) Conversely, if $\frac{a}{b}$ and $\frac{c}{d}$ are neighbors in a Farey sequence the next higher order Farey element appearing in between is $\frac{a+c}{b+d}$. For example, the next term appearing between $\frac{0}{1}$ and $\frac{1}{5}$ of $F_{5}$ is $\frac{0+1}{1+5}=\frac{1}{6}$ belonging to $F_{6}$. (iv) If $\frac{a}{b}$ and $\frac{c}{d}$ are neighbors in a Farey sequence of order $N$, the next term in the same sequence is given by the following expression:

$$
\frac{\left\lfloor\frac{N+b}{d}\right\rfloor c-a}{\left\lfloor\frac{N+b}{d}\right\rfloor d-b},
$$

where $\lfloor x\rfloor$ is the floor function. Therefore, the Farey sequence of order $N$ can be generated very efficiently starting from the elements $\frac{a}{b}=\frac{0}{1}$ and $\frac{c}{d}=\frac{1}{N}$.

In the next section the resonance sequence is introduced to describe the popular two-dimensional resonance diagram. A relation formula derived from the Farey sequence properties is obtained to efficiently generate all twodimensional resonances.

\section{RESONANCE SEQUENCES}

In accelerator physics the tune resonance condition in two dimensions is given by [3]

$$
a Q_{x}+b Q_{y}=c
$$

$a, b$, and $c$ being integers and the order of the resonance being $N=|a|+|b|$. To have a unique representation of a given resonance line in the tune diagram, Eq. (4) should be divided by the greatest common divisor of $a, b$, and $c$. In the following $|a|,|b|$, and $|c|$ are assumed to be coprime numbers and $a$ is chosen to be positive.

The tune resonance lines up to order 5 which traverse the square delimited by the points $\left(Q_{x}, Q_{y}\right)=$ $\{(0,0),(0,1),(1,1),(1,0)\}$ (the unitary square) are graphically represented in Fig. 1. Although much less obvious, the resonance lines in the diagram are still tightly related to Farey sequences. 


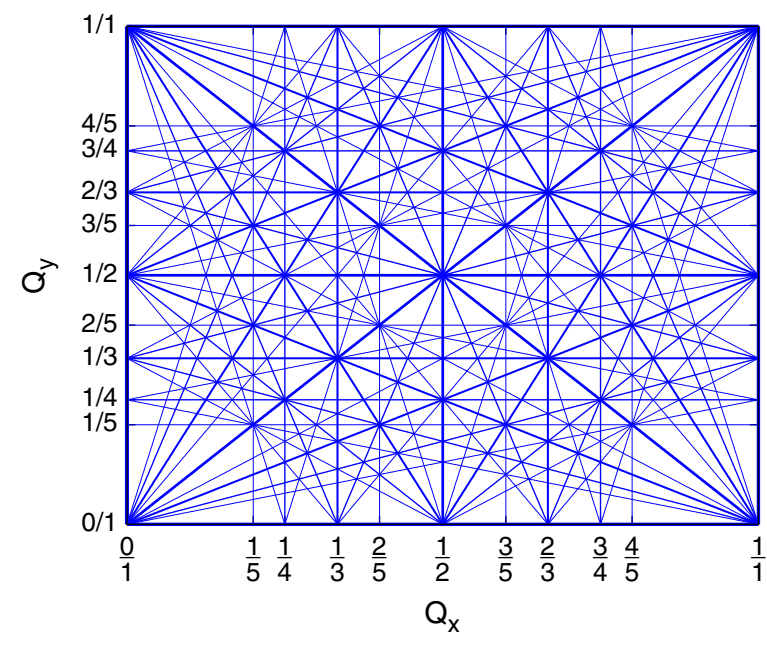

FIG. 1 (color online). Tune diagram displaying all resonances up to order 5 in the unitary square.

The resonance lines cut the horizontal axis at the Farey fractions. The bundle of resonance lines of order $N$ or lower converging in the point $\left(Q_{x}, Q_{y}\right)=(h / k, 0)$ (being $h / k$ a Farey fraction) consists of lines as Eq. (4) with $a,|b|$, and $|c|$ positive coprime integers fulfilling that

$$
\begin{aligned}
a \frac{h}{k} & =c, \\
a+|b| & \leq N .
\end{aligned}
$$

This implies that $c$ is a positive integer and that $a$ is a multiple of $k$. Let $a=k p$, then

$$
\begin{aligned}
p h & =c, \\
p k+|b| & \leq N .
\end{aligned}
$$

Subtracting $|b|$ to both sides of Eq. (6) and dividing by $p$,

$$
\frac{1}{k} \geq \frac{p}{N-|b|},
$$

this equation is fulfilled for $p /(N-|b|)$ being any of the Farey fractions of order $N$ below or equal $1 / k$. These solutions represent a truncated Farey sequence extending between $0 / 1$ and $1 / k$. Let this truncated Farey sequence be represented by $F_{N}^{1 / k}$.

For convenience the resonance sequence of order $N$ attached to the Farey fraction $\frac{h}{k}$ is defined as the set of pairs

$$
(a, b)=(k p, q-k p),
$$

where $p / q$ is an element of $F_{N}$ and $p / q \leq 1 / k$. This sequence is generated very similarly to Farey sequences starting from the elements $(a, b)=(0,1)$ and $(c, d)=$ $(k, N-k)$ and computing iteratively the next term with the following expression:

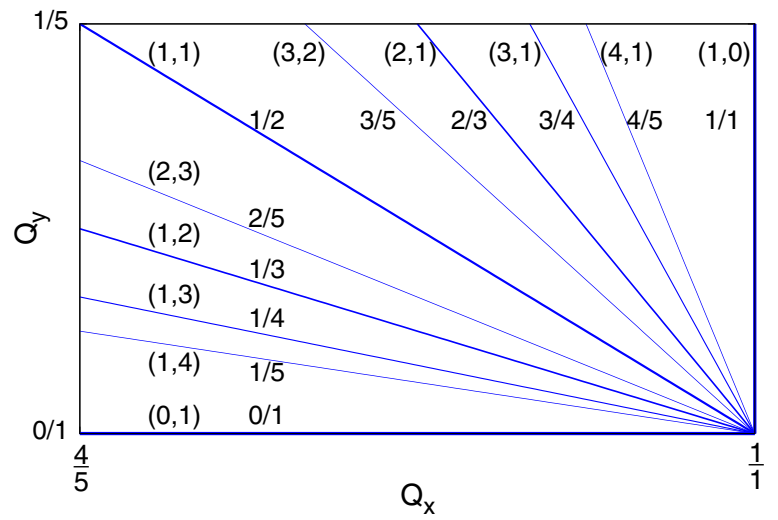

FIG. 2 (color online). Graphical representation of the resonance sequence of order 5 attached to $1 / 1$. The resonance lines are represented by $(a, b)$ and the corresponding Farey fraction by $p / q$. Note that $(a, b)=(p, q-p)$. The free term $c$ to complete the resonance equation is $c=a$.

$$
\left(\left\lfloor\frac{N+b+a}{d}\right\rfloor c-a,\left\lfloor\frac{N+b+a}{d}\right\rfloor d-b\right) .
$$

For example, the resonance sequence of order 5 attached to $1 / 1$ is given by

$$
\begin{gathered}
R_{5}^{1 / 1}=\{(0,1),(1,4),(1,3),(1,2),(2,3),(1,1), \\
(3,2),(2,1),(3,1),(4,1),(1,0)\} .
\end{gathered}
$$

Note that the corresponding properties (ii) and (iii) of Farey sequences given in Sec. I also exist for resonance sequences. Figures 2 and 3 show the graphical representations of the resonance sequences $R_{5}^{1 / 1}$ and $R_{5}^{1 / 2}$ in the tune diagram. $R_{5}^{1 / 1}$ contains all resonance lines up to order 5 that meet at $\left(Q_{x}, Q_{y}\right)=(1 / 1,0) . R_{5}^{1 / 2}$ contains the resonance lines up to order 5 coming from the left and meeting at

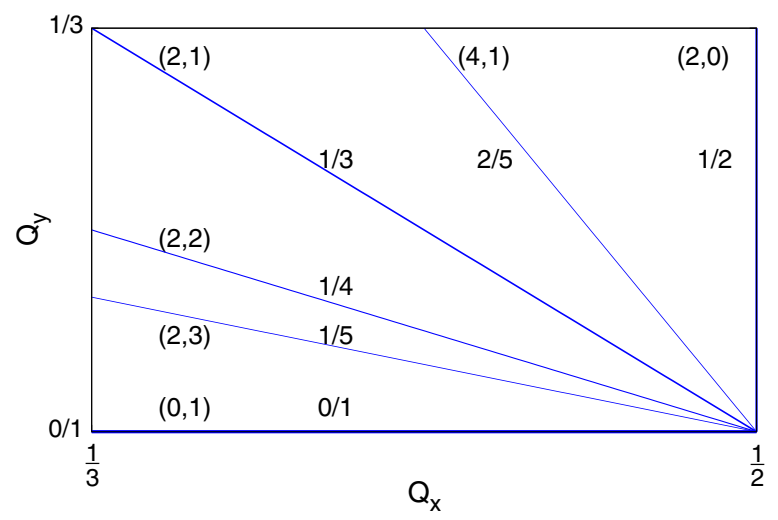

FIG. 3 (color online). Graphical representation of the resonance sequence of order 5 attached to $1 / 2$. The resonance lines are represented by $(a, b)$ and the corresponding Farey fraction by $p / q$. Note that $(a, b)=(2 p, q-2 p)$. The free term $c$ to complete the resonance equation is $c=a / 2$. 
$\left(Q_{x}, Q_{y}\right)=(1 / 2,0)$. The corresponding resonance lines coming from the right are mirror symmetric about $Q_{x}=1 / 2$, i.e., $(a,-|b|)$. The free term $c$ to complete the resonance Eq. (4) is just $c=a h / k$. Once the resonance lines crossing the horizontal axis have been generated, it is trivial to compute all resonance lines in the unitary square using the symmetries of the problem.

Thanks to these properties resonance diagrams can be generated and explored in the most efficient way. Moreover, it is possible to count the number of resonance lines up to order $N$ within the unitary square, $T_{N}$, using the following expression:

$$
T_{N}=4-2\left(4\left|F_{N}\right|-6\right)+\sum_{h / k \in F_{N}^{1 / 2}} 2\left(4\left|F_{N}^{1 / k}\right|-6\right),
$$

where the first monomial 4 accounts for the resonance lines on the sides of the unitary square $\left(T_{0}=4\right)$, the sum extends over all the Farey fractions $h / k$ in $F_{N}^{1 / 2}$ (it is not needed to sum over all fractions in $F_{N}$ thanks to the symmetry about $\frac{1}{2}$ ), and $\left|F_{N}\right|$ and $\left|F_{N}^{1 / k}\right|$ represent the number of elements in $F_{N}$ and $F_{N}^{1 / k}$, respectively. Note that the second monomial, $-2\left(4\left|F_{N}\right|-6\right)$, cancels the first term of the summation, $2\left(4\left|F_{N}^{1 / 1}\right|-6\right)$. Since $\left|F_{N}\right|$ tends asymptotically to $3 N^{2} / \pi^{2}, T_{N}$ is expected to grow with the third power of $N$ and a fit over the first 3000 values gives $T_{N} \approx 3.7+0.5547 N^{3}$ with a largest relative deviation below $9 \%$.

\section{CONCLUSIONS}

Resonance lines in the unitary square are fully described using Farey sequences. This leads into the definition of resonance sequences which share similar properties with the Farey sequences including a relation to very efficiently generate these sequences.

\section{ACKNOWLEDGMENTS}

I am very thankful to Andrea Franchi for proofreading the manuscript and making valuable suggestions.

[1] G. H. Hardy and E. M. Wright, An Introduction to the Theory of Numbers (Oxford Science Publications, New York, 1996), 5th ed.

[2] H. Brown and K. Mahler, J. Number Theory 3, 364 (1971).

[3] E. D. Courant and H. S. Snyder, Ann. Phys. (N.Y.) 281, 360 (2000); Ann. Phys. (N.Y.) 3, 1 (1958). 